Hans Pottgießer

\title{
Sicher auf den Schienen
}

Fragen zur Sicherheitsstrategie der Eisenbahn von 1825 bis heute

Springer Basel AG 
CIP-Titelaufnahme der Deutschen Bibliothek

\section{Pottgießer, Hans:}

Sicher auf den Schienen : Fragen zur Sicherheitsstrategie

d. Eisenbahn von 1825 bis heute / Hans Pottgießer.

\section{ISBN 978-3-0348-5257-9}

DOI 10.1007/978-3-0348-5256-2

ISBN 978-3-0348-5256-2 (eBook)

Das Werk ist urheberrechtlich geschützt. Die dadurch begründeten Rechte, insbesondere die der Ubersetzung, des Nachdruckes, der Entnahme von Abbildungen, der Funksendung, der Wiedergabe auf photomechanischem oder ähnlichem Wege und der Speicherung in Datenverarbeitungsanlagen bleiben, auch bei nur auszugsweiser Verwertung, vorbehalten. Die Vergütungsansprüche des $\S 54$, Abs. 2 UrhG werden durch die (Verwertungsgesellschaft Wort〉, München, wahrgenommen.

(C) 1988 Springer Basel AG

Ursprünglich erschienen bei Birkhäuser Verlag Basel 1988

Softcover reprint of the hardcover lst edition 1988

Buchgestaltung: Albert Gomm swb/asg, Basel 
Dieses Buch ist all denen gewidmet,

die für die Sicherheit der Eisenbahn gedacht, geschaffen, gestritten haben. 
«Wahrlich, es ist nicht das Wissen, sondern das Lernen, nicht das Besitzen, sondern das Erwerben, nicht das Da-seyn, sondern das Hinkommen, was den größten Genuß gewährt.»)

Prof. Carl Friedrich Gauß (1777-1855) 


\section{Inhalt}

1 Sicherheitsstrategie, was heißt das? 6

2 Was ist eigentlich eine Eisenbahn? 9

3 Wie regelt und steuert man den Zugverkehr? 13

4 Wann darf ein Zug abfahren? 21

5 Wie macht sich ein Zug bemerkbar? 29

6 Und wie verständigt man sich über die Zugfolge? 33

7 Was überwand die optische Telegraphie? 39

8 Wie deckt man Gefahrstellen im Gleis? 54

9 Falsche Weichenstellung? Wie wird sie vermieden? 70

10 Ein sicherer Abstand. Kann man ihn garantieren? 85

11 Was ist ein Streckenblock? 93

12 Kann man Fehler bei der Blockbedienung ausschließen? 107

13 Wie verhindert man Gegenfahrten auf eingleisiger Strecke? 116

14 Wer führt das Kommando im Bahnhof? 127

15 Und was geschieht bei Nacht und Nebel? 137

16 Welche Signalfarben sind optimal? 146

17 Was bringt die elektro-mechanische Stellwerkstechnik? 155

18 Ist der Fahrweg frei? 166

19 Was stoppt den Zug am Haltsignal? 175

20 Wie vermeidet man zu schnelles Fahren? 190

21 Steigert die Relaistechnik die Sicherheit? 205

22 Wird die Sicherheit durch Signalstörungen beeinflußt? 217

23 Verbesserungen der Signaltechnik, wie schnell greifen sie? 225

24 Und was darf die Sicherheit kosten? 234

25 Bringt die Zukunft noch mehr Sicherheit? 238

26 Und wo bleibt der Mensch? 250

Anmerkungen $\quad 255$

Literaturhinweise $\quad 257$

$\begin{array}{lr}\text { Bildquellen } & 261\end{array}$ 